\title{
Management of Lytic Bone Disease in Lymphoplasmacytic Lymphoma: A Case Report and Review of the Literature
}

\author{
Mizba Baksh ${ }^{1}$, Liuyan Jiang ${ }^{1}$, Unnati Bhatia ${ }^{2}$, Victoria Alegria ${ }^{1}$, Taimur Sher ${ }^{1}$, Vivek \\ Roy $^{1}$, Asher Chanan-Khan ${ }^{1}$, Sikander Ailawadhi ${ }^{1}$, and Ricardo Parrondo ${ }^{1}$ \\ ${ }^{1}$ Mayo Clinic Hospital Jacksonville \\ ${ }^{2}$ Maulana Azad Medical College
}

July 10, 2021

\begin{abstract}
Waldenström Macroglobulinemia (WM)/lymphoplasmacytic lymphoma (LPL) is often differentiated from myeloma based on the presence of lytic bone lesions (LBL). However, WM/LPL can present with LBL and management is poorly understood. We describe a case of an 81-year-old woman with LPL who presented with LBL and was successfully treated with chemoimmunotherapy.
\end{abstract}

Management of Lytic Bone Disease in Lymphoplasmacytic Lymphoma: A Case Report and Review of the Literature

Mizba Baksh, M.B.B.S ${ }^{1}$, Liuyan Jiang, MD $^{2}$, Unnati Bhatia, M.B.B.S ${ }^{1}$, Victoria Alegria, ARNP ${ }^{1}$, Taimur Sher, $\mathrm{MD}^{1}$, Vivek Roy, $\mathrm{MD}^{1}$, Asher Chanan-Khan, $\mathrm{MD}^{1,3,4}$, Sikander Ailawadhi, MD ${ }^{1,3}$, Ricardo D. Parrondo, $\mathrm{MD}^{* 1}$

${ }^{1}$ Division of Hematology-Oncology, Mayo Clinic, Jacksonville, Florida, USA

${ }^{2}$ Department of Pathology, Mayo Clinic, Jacksonville, Florida USA

${ }^{3}$ Department of Cancer Biology, Mayo Clinic, Jacksonville, Florida, USA

${ }^{4}$ Hematology-Oncology, St. Vincent's Riverside, Jacksonville, Florida, USA

${ }^{*}$ Corresponding author:

Sikander Ailawadhi, MD

4500 San Pablo Road S

Mangurian Building, 3rd Floor

Jacksonville, Florida 32224

Ailawadhi.Sikander@mayo.edu

Abstract

Waldenström Macroglobulinemia (WM)/lymphoplasmacytic lymphoma (LPL) is often differentiated from myeloma based on the presence of lytic bone lesions (LBL). However, WM/LPL can present with LBL and 
management is poorly understood. We describe a case of an 81-year-old woman with LPL who presented with LBL and was successfully treated with chemoimmunotherapy.

\section{Key clinical message}

We have discussed how optimal therapeutic management of WM patients with lytic bone disease is poorly understood which necessitates the need for further investigation on the ability of novel agents and antiresorptive agents to reverse bone turnover in WM/LPL patients.

\section{Introduction}

Waldenström macroglobulinemia (WM) is a rare, indolent, lymphoproliferative disorder that represents $1 \%$ to $2 \%$ of all non-Hodgkin lymphomas (NHL) [1]. It is pathologically defined as lymphoplasmacytic lymphoma (LPL) by the World Health Organization and is characterized by bone marrow infiltration with clonal lymphoplasmacytic cells and IgM monoclonal gammopathy, although non-IgM secreting lymphoplasmacytic lymphomas have also been described $[2,3]$. Lytic bone lesions are rare in WM/LPL and are often used a differentiating clinical feature between WM/LPL and multiple myeloma (MM), particularly IgM myeloma. In a large study series of 37 patients, Schuster et al. used strict defining criteria for IgM myeloma to make a clear distinction from WM/LPL since the approach to their treatment and prognosis varies significantly. The inclusion criteria considered were the presence of IgM monoclonal protein and [?]10\% plasma cells in the bone marrow biopsy in addition to the characteristic lytic bone lesions with or without the most common cytogenetic abnormality of IgM myeloma i.e., translocation $t(11 ; 14)$. This study did not include patients based on non-specific clinical features of myeloma such as the presence of anemia, hypercalcemia, and renal failure or their immunophenotype [4-7]. However, lytic bone lesions in WM/LPL have been reported with little guidance on management. Rothschild et al documented in their clinical case study that WM/LPL has a combination of features of other hematologic malignancies such as myeloma and leukemia on both macroscopic and radiologic examination of osteolytic lesions. The lytic lesions of WM were either sharp spheroid lesions with effaced edges or abundant coalescing holes/pits with smooth edges and were identifiable from the numerous frontally resorptive non-spheroid leukemic lesions and the pit less although spheroid lesions of MM [8]. This is in contrast with MM, which tends to show four different forms of destructive bone changes on imaging studies- single expansile plasmacytoma, disseminated punched-out lytic lesions, diffuse skeletal osteopenia, or osteosclerosis [9, 10]. However, the biology of these differences in bone lesions between MM and WM/LPL are poorly understood. In a study by Papanikolaou et al, focal lytic bone disease was evident in 17 to $24 \%$ of WM cases when investigated retrospectively using either MRI (magnetic resonance imaging) or PET-CT (positron emission tomography-computed tomography) imaging, respectively [11]. Consequently, multiple studies have substantiated this rather unusual presentation in WM/LPL, while some even reported improvement of lytic lesions with treatment [12-16].

There is a paucity in the literature as to whether patients with WM/LPL and lytic bone lesions should be treated with chemoimmunotherapy or novel agents and whether bone strengthening agents should be used. A consensus panel from the 10th International Workshop on WM has updated both first line and salvage treatment recommendations. The preferred primary therapy options for symptomatic patients with WM include chemoimmunotherapeutic combination regimens of rituximab with alkylating agents (i.e., bendamustine, cyclophosphamide) and proteasome inhibitors (i.e., bortezomib) or with Bruton's tyrosine kinase (BTK) inhibitors like ibrutinib. Treatment options need to be customized according to the individual patient's clinical presentation and genomic features [17-20]. Studies for antiresorptive agents in WM/LPL are lacking, albeit many preclinical and randomized control studies of bisphosphonates and RANKL (receptor activator of nuclear factor-kappa B ligand) inhibitors in MM have demonstrated not only reduction of bone complications but potential anti-MM effects as well [21]. Herein we describe a case of LPL with lytic bone lesions who was treated with rituximab, cyclophosphamide, and dexamethasone and had achieved a CR with complete resolution of lytic bone lesions on PET-CT.

Case 
An 81-year-old woman with progressively deteriorating Parkinson's disease despite ongoing medical treatment for more than 5 years as well as osteoporosis (on denosumab q6 months) presented to our institution's spine center for worsening back pain and frequent falls. She was initially diagnosed with a mild degenerative disc disease of her cervical, thoracic, and lumbar spine without myelopathy as well as severe facet arthrosis in the lumbar spine from L2-3 through L5-S1 as demonstrated on MRI images. Due to progressive pain and the possibility of compression fractures from her recurrent falls, a PET-CT was performed which reported several foci of marked hypermetabolism including a dominant lesion in the right humeral head, SUV max 9.9 and additional hypermetabolic lesions were seen in the bilateral scapulae, clavicles, right hemi-sacrum, right iliac wing, left acetabulum anterior column, left superior pubic ramus, and right femoral head (Figure 1A ). The images were suggestive of MM-associated lytic lesions and a subsequent serum protein electrophoresis and immunofixation revealed small monoclonal IgA lambda immunoglobulins. Complete blood cell count, quantitative serum free light chains, $\beta 2$-microglobulin, albumin, $\mathrm{LDH}$ and creatinine were all within normal ranges. The patient did have mild hypercalcemia with a calcium level of $10.3 \mathrm{mg} / \mathrm{dL}$. A subsequent bone biopsy from the left anterior acetabulum was obtained, which revealed diffuse proliferation of small Blymphocytes with an interstitial and para-trabecular pattern (Figure 2A ). Immunohistochemistry (IHC) studies showed that the lymphocytes were positive for CD20 and PAX5 (Figure 2 B and C ), which proved the B cell lineage of the lymphoma. The neoplastic lymphocytes were negative for MUM1 and cyclin D1. Moreover, CD138 highlighted scattered plasma cells that were positive for IgA and lambda light chain restricted (Figure 2D-F ). A MYD88 L265P alteration was detected via amplification of DNA using allelespecific polymerase chain reaction with an allele-specific primer. These findings were most consistent with a lymphoplasmacytic lymphoma.

Given the diagnosis of lymphoplasmacytic lymphoma in a frail patient with advanced Parkinson's disease and an ECOG (Eastern Cooperative Oncology Group) performance status of 2, it was decided to treat the patient with chemoimmunotherapy consisting of IV rituximab $375 \mathrm{mg} / \mathrm{m}^{2}$, IV cyclophosphamide $300 \mathrm{mg} / \mathrm{m}^{2}$ and IV dexamethasone $20 \mathrm{mg}$ given every 21 days. The patient completed 6 cycles of treatment. Treatment was complicated by the development of grade 2 neutropenia and grade 1 anemia. The patient also developed a grade 2 urinary tract infection that needed treatment with oral antibiotics and grade 2 herpes labialis which required acyclovir treatment for 10 days after cycle 4 causing cycle 5 to be delayed by 1 week. Following completion of the 6 cycles of treatment, the patient had complete resolution of lytic lesions on PET-CT (Figure 1B) and no detectable IgA kappa monoclonal protein on immunofixation. The patient went on to start maintenance therapy with single agent rituximab every 3 months for up to 2 years. The patient still had some back pain which is likely related to her underlying degenerative joint disease and severe facet arthrosis in the lumbar spine so she will follow-up with neuroradiology for possible facet joint injections and/or potential kyphoplasty.

\section{Discussion}

We described a case of an IgA lambda, MYD88L265 mutation+ lymphoplasmacytic lymphoma with lytic bone lesions that was successfully treated with rituximab, cyclophosphamide and dexamethasone resulting in a complete response. There is scarce literature on optimal treatment for patients with WM/LPL and lytic bone disease. A multitude of novel agents are approved for the treatment of WM/LPL such as BTK inhibitors, proteasome inhibitors and monoclonal antibodies and many more next-generation therapies in these drug classes are under development in addition to BCL2- inhibitors like venetoclax and phosphatidylinositol 3 kinase inhibitors such as idelalisib and umbralisib [22]. However, the efficacy of these agents in WM/LPL patients with lytic bone disease is unknown. The efficacy of agents such as proteasome inhibitors, immunomodulatory drugs, and alkylating agents on bone remodeling in MM is well established [23]. In lymphoid malignancies like chronic lymphocytic leukemia (CLL), only BTK inhibitors like ibrutinib have shown promising therapeutic response in patients with osteolytic lesions [24-26]. There is evidence that the BTK inhibitor ibrutinib can suppress bone resorption by inhibition of both osteoclast differentiation and function, predominantly by downregulation of expression of Nuclear Factor Of Activated T Cells 1 (NFATc1), the key transcription factor for osteoclastogenesis, and disruption of the formation of the actin ring in mature osteoclasts [27]. In one case of an elderly woman with relapsed CLL/SLL (chronic lymphocytic leukemia/small 
lymphocytic lymphoma) with widespread lytic disease and pathological fractures, treatment with ibrutinib monotherapy (420 mg q.d.) with monthly denosumab (120 mg s.c.) for only 9 months resulted in remineralization of her skeletal lesions and partial disease response. The combination of a BTK Inhibitors with a bone-resorptive agent provided significant clinical benefit with remarkable improvement in patient mobilization after about 12 months of treatment with sclerosis of skeletal lesions as noted on serial CT and MRI scans [28].

The modulation of bone remodeling by anti-myeloma agents such as immunomodulatory drugs, proteasome inhibitors and monoclonal antibodies provide insight into their potential efficacy and mechanism of action in patients with WM/LPL [29]. Via interactions with the bone marrow microenvironment, malignant plasma cells are able to orchestrate the production of osteoclast-activating factors (i.e. RANKL) and osteoblast-inhibitory factors which leads to asynchronous bone turnover, net bone loss and osteolytic lesions. Proteasome inhibitors such as bortezomib, carfilzomib, and ixazomib inhibit NF- $\varkappa \mathrm{B}$ (nuclear factor kappa-B) mediated osteoclast maturation and ultimately, bone resorption via the RANKL and OPG (osteoprotegerin) pathway [30]. Terpos et al demonstrated that bortezomib also increased bone formation markers like bonespecific alkaline phosphatase (ALP) and osteocalcin levels with only four cycles of treatment in 34 relapsed MM patients [21]. Furthermore, bortezomib has shown inhibition of osteoclastogenesis in combination with the immunomodulatory drug lenalidomide in vitro. [31]. Both of these agents have also shown the ability to reduce tumor burden in MM patients through their inhibitory effect on osteoclast-derived growth and survival factors and blocking of RANKL secretion from bone marrow stromal cells [31, 32]. Lenalidomide inhibits osteoclastogenesis as evidencde by decreased serum biochemical markers of bone turnover [32]. Pomalidomide, another immunomodulatory drug, has shown potent osteoclast inhibitory activity in vitro with its downregulating effect on transcription factor PU.1 and significant blunting of RANKL upregulation, thus normalizing the RANKL-OPG ratio [33]. Daratumumab, an anti-CD38 monoclonal antibody inhibits bone remodeling by blocking the interaction of CD38 expressing monocytes and osteoclast-progenitor cells thus inhibiting bone resorption activity in bone marrow cells of MM patients [34, 35]. In a study of $51 \mathrm{MM}$ patients, high dose chemotherapy with melphalan followed by autologous stem cell transplant (ASCT) resulted in a significant reduction of sRANKL/OPG ratio, with a concomitant decrease in markers of bone resorption starting the second month post-ASCT[36]. Further investigation is needed on whether these active anti-MM agents have similar effects on bone turnover in patients with non-Hodgkin lymphomas such as WM/LPL with lytic bone lesions.

Although chemoimmunotherapy combinations are current standard treatment regimens and are highly active with high response rates, they can cause immunosuppression and cytopenias which may not be well tolerated by elderly, frail patients. With a median age of diagnosis of WM/LPL being 70 years, consideration must be given to patient frailty and ability to tolerate such a treatment. However, cyclophosphamide is well tolerated in elderly patients when used as a combination regimen with rituximab and dexamethasone (DRC). This was demonstrated in a study conducted by Dimopolous et al in a large multicenter trial of 72 patients with WM, whose median age was 69 years and among which $63 \%$ patients were older than 65 years old. Based on analysis of this study, therapy with DRC was well tolerated and only about $10 \%$ of patients experienced grade 3 or 4 neutropenia, and $10 \%$ of patients developed neutropenic fever requiring hospitalization and intravenous antibiotics. No patients developed grade 3 or 4 thrombocytopenia. Therefore, DRC is a safe and well-tolerated regimen, even in elderly frail patients [37]. The DRC regimen was also used successfully in a 64-year-old patient who was diagnosed with WM and had mixed lytic and sclerotic lesions on skeletal radiographs and CT scans. The patient tolerated 6 cycles of DRC treatment with no significant toxicity or signs of lymphoma progression after a follow up of 32 months. The majority of the patient's bone lesions also disappeared with treatment except for one persistent bone lesion which was treated with 8Gy of radiation therapy [38]. Based on the available data, including our case report, DRC is an efficacious regimen for patients with WM and lytic bone lesions.

Interestingly, our patient was on denosumab every 6 months for osteoporosis, but there are no consensus guidelines on the use of antiresorptive agents for patients with WM/LPL and lytic bone lesions. Most of the literature and evidence comes from the treatment of MM as previously described [21]. The risk of high bone 
turnover and premature osteoporosis in lymphoma patients due to treatment with high dose corticosteroids can be counteracted by the prophylactic use of antiresorptive agents. In patients with lymphoma receiving chemotherapy, treatment with the second-generation bisphosphonate pamidronate every 3 months for 1 year reduced both bone loss and the risk of new vertebral fractures [39]. A prospective randomized phase III trial investigated the benefit of using zoledronic acid (ZA) in 74 newly diagnosed lymphoma patients undergoing chemotherapy and with a baseline bone mineral density (BMD) of [?] -2.0. A dose of 4mg IV ZA was given at trial enrollment and at 6 months along with oral calcium $(1200 \mathrm{mg})$ and vitamin D (400 or $800 \mathrm{IU})$. Fifty-three patients were evaluable for response: 24 received ZA and had stable BMD during the observation period, whereas 29 patients in the control group had decreased BMD $(\mathrm{P}<0.05$ at lumbar spine and bilateral femoral neck) [40]. Further investigation into use of antiresorptive agents for patients with WM/LPL and lytic bone lesions is warranted.

\section{Conclusion}

Lytic bone lesions associated with non-Hodgkin's lymphomas such as CLL and WM/LPL are well-reported in the literature. However, the biology of these bone lesions is poorly understood as is the optimal therapeutic management of patients with lytic bone disease. Drugs such as immunomodulatory agents, proteasome inhibitors, monoclonal antibodies, and high dose melphalan followed by autologous stem cell transplant have been extensively proven to reduce bone resorption in MM. Furthermore, the addition of antiresorptive agents such as denosumab or ZA prevent bone resorption and subsequent fractures in patients with MM. Many of these agents have efficacy in WM/LPL yet their role in WM patients with lytic bone lesions is unknown. Our case demonstrates the efficacy of the chemoimmunotherapy regimen DRC in causing a complete response with resolution of lytic bone lesions in a patient with LPL. Further research is warranted on the ability of novel agents to reverse bone turnover in WM/LPL patients as well as on the utility on antiresorptive agents in non-Hodgkin's lymphomas with lytic bone disease.

\section{Conflict of Interest}

M.B, R.D.P, U.B. L.J, V.A, T.S, and A. C-K have no conflicts of interest to declare.

S.A receives honoraria from Celgene and Takeda as well as research funding from Amgen, Janssen, Pharmacyclics, Cellectar, Bristol Myers Squibb, Medimmune and Phosplatin.

\section{Authors' Contributions}

M.B. and R.D.P: wrote the manuscript; LJ: obtained the pathology images for the case; M.B. R.D.P U.B., V.A, V.R., T.S, A.C-K, and S.A edited and finalized the manuscript.

\section{References}

1. Sekhar, J., et al., Waldenström macroglobulinemia: a Surveillance, Epidemiology, and End Results database review from 1988 to 2005. Leuk Lymphoma, 2012. 53 (8): p. 1625-6.

2. Campo, E., et al., The 2008 WHO classification of lymphoid neoplasms and beyond: evolving concepts and practical applications.Blood, 2011. 117 (19): p. 5019-5032.

3. Owen, R.G., et al., Clinicopathological definition of Waldenstrom's macroglobulinemia: consensus panel recommendations from the Second International Workshop on Waldenstrom's Macroglobulinemia.Semin Oncol, 2003. 30 (2): p. 110-5.

4. Schuster, S.R., et al., IgM multiple myeloma: Disease definition, prognosis, and differentiation from Waldenstrom's macroglobulinemia. American Journal of Hematology, 2010.85 (11): p. 853-855.

5. Feyler, S., et al., IgM myeloma: a rare entity characterized by a CD20-CD56-CD117- immunophenotype and the t(11;14). Br J Haematol, 2008. 140 (5): p. 547-51. 
6. Avet-Loiseau, H., et al., $14 q 32$ Translocations discriminate IgM multiple myeloma from Waldenstrom's macroglobulinemia. Semin Oncol, 2003. 30 (2): p. 153-5.

7. Bonilla-Valentín, F.J., et al., Case Report of IgM Multiple Myeloma: Diagnosing a Rare Hematologic Entity. Cancer Control, 2018.25 (1): p. 1073274817744448.

8. Rothschild, B.M., F. Ruhli, and C. Rothschild, Skeletal clues apparently distinguishing Waldenstrom's macroglobulinemia from multiple myeloma and leukemia. American Journal of Human Biology, 2002.14 (4): p. $532-537$.

9. Angtuaco, E.J., et al., Multiple myeloma: clinical review and diagnostic imaging. Radiology, 2004. 231 (1): p. 11-23.

10. Hanrahan, C.J., C.R. Christensen, and J.R. Crim, Current concepts in the evaluation of multiple myeloma with MR imaging and FDG PET/CT. Radiographics, 2010. 30 (1): p. 127-42.

11. Papanikolaou, X., et al., Waldenstrom's Macroglobulinemia Associated Bone Disease the UAMS Experience. Blood, 2014.124 (21): p. 2999-2999.

12. Marks, M.A., D.E. Tow, and M. Jay, Bone scanning in Waldenstrom's macroglobulinemia. Journal of nuclear medicine : official publication, Society of Nuclear Medicine, 1985. 26 (12): p. 1412-1414.

13. Banwait, R., et al., Extramedullary Waldenström macroglobulinemia. American Journal of Hematology, 2015.90 (2): p. 100-104.

14. Treon, S.P., et al., Ibrutinib in Previously Treated Waldenström's Macroglobulinemia. New England Journal of Medicine, 2015.372 (15): p. 1430-1440.

15. Mehmood, K., et al., Waldenstroms Macroglobulinemia Patient Presenting with Rare 'lytic' Lesions and Hypercalcemia: A Diagnostic Dilemma. J Clin Diagn Res, 2014. 8 (11): p. Fd10-1.

16. Pujani, M., et al., Waldenstrom's macroglobulinemia presenting with lytic bone lesions: A rare presentation. Blood research, 2013.48 : p. 230-233.

17. Castillo, J.J., et al., Consensus treatment recommendations from the tenth International Workshop for Waldenström Macroglobulinaemia. Lancet Haematol, 2020. 7 (11): p. e827-e837.

18. Dimopoulos, M.A., et al., Waldenström's macroglobulinemia: clinical features, complications, and management. J Clin Oncol, 2000.18 (1): p. 214-26.

19. Dimopoulos, M.A., et al., Update on treatment recommendations from the Fourth International Workshop on Waldenstrom's Macroglobulinemia. J Clin Oncol, 2009. 27 (1): p. 120-6.

20. Treon, S.P., et al., Novel agents in the treatment of Waldenström's macroglobulinemia. Clin Lymphoma Myeloma, 2007. 7 Suppl 5 : p. S199-206.

21. Terpos, E., et al., Treatment of multiple myeloma-related bone disease: recommendations from the Bone Working Group of the International Myeloma Working Group. Lancet Oncol, 2021.22 (3): p. e119-e130.

22. Castillo, J.J. and S.P. Treon, What is new in the treatment of Waldenstrom macroglobulinemia? Leukemia, 2019. 33 (11): p. 2555-2562.

23. Raje, N. and G.D. Roodman, Advances in the Biology and Treatment of Bone Disease in Multiple Myeloma. Clinical Cancer Research, 2011. 17 (6): p. 1278-1286.

24. Dimopoulos, M.A., et al., Ibrutinib for patients with rituximab-refractory Waldenström's macroglobulinaemia (iNNOVATE): an open-label substudy of an international, multicentre, phase 3 trial.Lancet Oncol, 2017. 18 (2): p. 241-250.

25. Shumilov, E., et al., Osteolytic lesions occur rarely in patients with B-CLL and may respond well to ibrutinib. Leukemia \& Lymphoma, 2016. 57 (10): p. 2476-2480. 
26. O'Brien, S., et al., Ibrutinib as initial therapy for elderly patients with chronic lymphocytic leukaemia or small lymphocytic lymphoma: an open-label, multicentre, phase 1b/2 trial. Lancet Oncol, 2014 . 15 (1): p. $48-58$.

27. Shinohara, M., et al., The orally available Btk inhibitor ibrutinib (PCI-32765) protects against osteoclastmediated bone loss.Bone, 2014. 60 : p. 8-15.

28. Tucker, D.L., et al., Remineralization of lytic bone disease in a patient with small lymphocytic lymphoma using ibrutinib. British Journal of Haematology, 2017.178 (1): p. 153-155.

29. Silbermann, R. and G.D. Roodman, Current Controversies in the Management of Myeloma Bone Disease. Journal of Cellular Physiology, 2016. 231 (11): p. 2374-2379.

30. Delgado-Calle, J., T. Bellido, and G.D. Roodman, Role of osteocytes in multiple myeloma bone disease. Curr Opin Support Palliat Care, 2014. 8 (4): p. 407-13.

31. Terpos, E., et al., The combination of intermediate doses of thalidomide with dexamethasone is an effective treatment for patients with refractory/relapsed multiple myeloma and normalizes abnormal bone remodeling, through the reduction of sRANKL/osteoprotegerin ratio.Leukemia, 2005.19 (11): p. 1969-1976.

32. Breitkreutz, I., et al., Lenalidomide inhibits osteoclastogenesis, survival factors and bone-remodeling markers in multiple myeloma. Leukemia, 2008. 22 (10): p. 1925-1932.

33. Anderson, G.l.m., et al., Thalidomide derivative CC-4047 inhibits osteoclast formation by down-regulation of PU.1. Blood, 2006.107 (8): p. 3098-3105.

34. Costa, F., et al., Expression of CD38 in myeloma bone niche: A rational basis for the use of anti-CD38 immunotherapy to inhibit osteoclast formation. Oncotarget, 2017. 8 (34): p. 56598-56611.

35. Moreau, P. and C. Touzeau, Multiple Myeloma: From Front-Line to Relapsed Therapies. American Society of Clinical Oncology Educational Book, 2015(35): p. e504-e511.

36. Terpos, E., et al., Autologous stem cell transplantation normalizes abnormal bone remodeling and sRANKL/osteoprotegerin ratio in patients with multiple myeloma. Leukemia, 2004.18 (8): p. 1420-1426.

37. Dimopoulos, M.A., et al., Primary Treatment of Waldenström Macroglobulinemia With Dexamethasone, Rituximab, and Cyclophosphamide.Journal of Clinical Oncology, 2007. 25 (22): p. 3344-3349.

38. Koehler, M., et al., Mixed Lytic and Blastic Bone Lesions as a Presenting Feature of Waldenström Macroglobulinemia: Case Report and Review of the Literature. Clinical Lymphoma Myeloma and Leukemia, 2020.20 (2): p. e87-e91.

39. Kim, S.H., S.K. Lim, and J.S. Hahn, Effect of pamidronate on new vertebral fractures and bone mineral density in patients with malignant lymphoma receiving chemotherapy. Am J Med, 2004.116 (8): p. 524-8.

40. Westin, J.R., et al., Zoledronic Acid for Prevention of Bone Loss in Patients Receiving Primary Therapy for Lymphomas: A Prospective, Randomized Controlled Phase III Trial. Clinical Lymphoma, Myeloma and Leukemia, 2013. 13 (2): p. 99-105. 

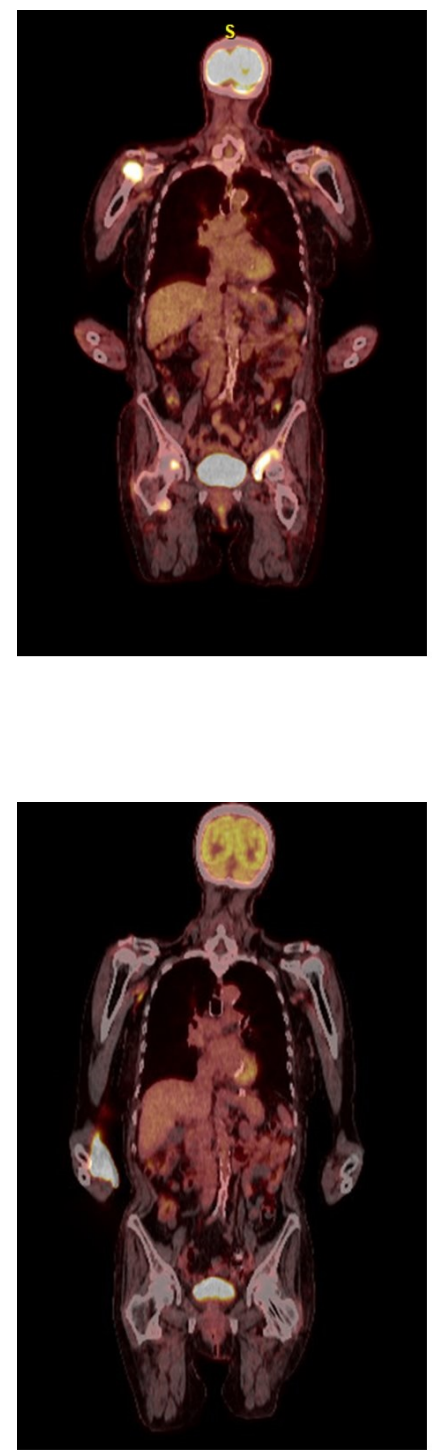


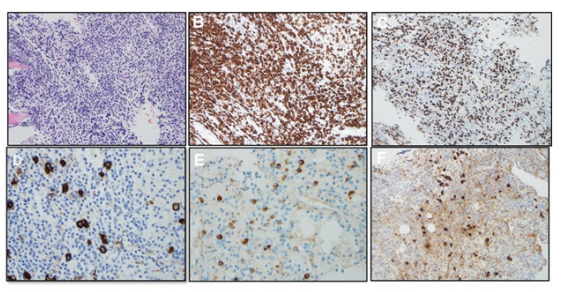

